\title{
Perspectives From the Assistant Secretary for Preparedness and Response
}

\author{
Nicole Lurie, MD, MSPH
}

W ith each disaster, whether an infectious disease outbreak or a natural disaster, we are reminded of the importance of having a health care system that is prepared to efficiently and effectively care for large numbers of sick or injured people. We've been building our national preparedness system for well over a decade and have seen significant progress, particularly in hospital preparedness. But our populations are changing, as is our health care system. People are living longer, and with advances in technology and modern medical care, are more able to live independently. By 2050, the number of individuals using paid long-term care services in any setting (eg, at home, residential care such as assisted living, or skilled nursing facilities) will likely double to 27 million people. ${ }^{1}$ Care is increasingly shifting from the acute care hospital to ambulatory settings-surgicenters, urgent care centers, dialysis facilities, and ambulatory care sites are all more prevalent than a decade ago. The Affordable Care Act has accelerated transformation of the delivery system; new models of care are emerging throughout the country. Health care facilities are consolidating and integrating, both horizontally and vertically, and networks of care for any given individual involve multiple kinds of facilities and health care professionals.

In recognition of these changes, the national Hospital Preparedness Program has also been evolving and has shifted from thinking about preparedness one hospital at a time to considering the preparedness of the entire health care system in a community. Health care entities in these communities are increasingly organized into health care coalitions for the purposes of enhancing emergency preparedness and response. The ongoing growth and development of health care coalitions will play an increasingly important role in the advancement of domestic health care system preparedness. Because health care coalitions are relatively new, evidence is scant about how to build them and get them to function optimally. This issue of Disaster Medicine and Public Health Preparedness aims to improve the evidence base to best support coalition development.

Health care coalitions have already demonstrated their value, and that begins by improving the relationship between public health and day-to-day health care. Two-thirds of public health departments are now involved in regional health care coalitions, fostering stronger population health networks, more robust health care infrastructure, and more resilient communities. Moreover, coalitions provide a forum for the integration of health care partners across the full spectrum of patient care, including long-term care, emergency medical services (EMS), outpatient centers, and home health agencies. For example, in South Carolina, one coalition has invested in an application that supports improved first responderto-first receiver communication in day-to-day emergency care as well as in disasters. The application can be used to track patients through the full spectrum of care, notify receiving facilities of patient transfer, provide clinical decision support, enable basic EMS telemedicine, and monitor emergency department status. Involvement in coalitions also builds community trust and enables health partners to access information and supplies when they are needed most. In Palm Beach County, Florida, coalition-driven networking and established memoranda of understanding have helped to mitigate and respond to "daily emergencies" and in disasters. Their diverse coalition membership has provided facilities in need with quick and easy access to blood products, saline, and pharmaceuticals in shortage; coalition members have donated emergency generators and essential medical equipment; pediatric hospitals have responded to member hospital inquiries about pediatric dosing of oseltamivir and countermeasure availability for radiological events; and, finally, pharmacy clinics have supported public education and immunization campaigns. Additional contributions of coalition activities to daily health care delivery are well described in this issue.

Furthermore, my office has been working to support regulations that provide comprehensive preparedness standards for a variety of providers and suppliers. This effort aims to fill a critical gap by ensuring that providers in every community not only are individually prepared in the event of an emergency or disaster but also have the training, plans, and policies needed to effectively connect to the rest of their community to deliver continuous, critical, and life-saving care. We are confident in coalitions' ability to provide this support and have made a number of resources 
available to assist in this endeavor: ASPR's Technical Resources Assistance Center and Information Exchange (TRACIE) provides access to information, technical experts, and resources that support strong and resilient coalitions and health care systems (https://asprtracie.hhs.gov/); the Emergency Care Coordination Center (ECCC) offers additional resources, with the goal of creating an emergency care system that is patient- and community-centered, integrated into the broader health care system, and prepared to respond in times of public health emergencies (www.phe.gov/ECCC); and the US Department of Health and Human Services emPOWER map overlays areas at risk for power outages with the monthly total of Medicare beneficiaries with electricity-dependent equipment claims in a given geographical area (http://www.phe.gov/empowermap).

As coalitions continue to mature and evolve, so too will their role in national health security. While they have already accomplished much, we must not grow complacent in our achievements. As we envision the next generation of coalitions, it is time to advance our focus away from planning and toward operations. The experience in Philadelphia following the May 12, 2015, Amtrak derailment highlights coalitions' operational role. On that day, disaster pre-planning helped 12 Philadelphia hospitals, including those from the Northeast and Southcentral Healthcare Coalitions, operationalize their communication platforms and patient tracking initiatives to quickly and safely move 100 patients to appropriate levels of hospital care. This experience also emphasizes the notion that in an emergency, we respond with the system capabilities that we use every day. Thus, improving the daily function of the health care system is central to health care preparedness. Given this reality, I challenge you to investigate how your own coalition's activities may best align with and support the augmentation of day-to-day capabilities and to think about how current health care reform initiatives can be better leveraged to promote resilient and prepared health care systems not only in disasters, but every single day. Meanwhile, I hope the articles in this issue can suggest practices to adopt and will also stimulate a research agenda for coalitions of the future.

\section{About the Author}

Office of the Assistant Secretary for Preparedness and Response, US Department of Health and Human Services, Washington, DC.

Correspondence and reprint requests to Nicole Lurie, 200 Independence Avenue SW, Room 639D, Washington DC 20201 (e-mail: nicole.lurie@hhs.gov).

\section{REFERENCE}

1. US Department of Health and Human Services, Centers for Disease Control and Prevention. Long-term care services in the United States: 2013 overview. Vital Health Stat. 2013;3(37). 